\title{
Segurança do paciente na emergência: compreensão da equipe de um hospital de referência
}

\author{
Patient safety in the emergency: understanding of the staff of a reference hospital \\ Seguridad del paciente en la emergencia: comprensión del personal de un hospital de referencia
}

\begin{abstract}
RESUMO
Objetivo: Compreender a percepção da equipe de enfermagem e médica que atua em uma unidade de emergência, quanto às metas de segurança do paciente. Método: Trata-se um estudo descritivo com abordagem qualitativa, realizado em um setor de emergência de um hospital público no Paraná. A coleta de dados foi realizada por meio de entrevistas guiadas por um roteiro, contendo questões abertas e fechadas. Resultados: Os participantes do estudo foram 15 profissionais dentre eles enfermeiros, médicos e técnicos de enfermagem, que destacaram sua visão e compreensão sobre as metas de segurança do paciente propostas pelo Ministério da Saúde, assim como, a aplicação diária dentro de sua prática assistencial. Conclusão: 0 estudo possibilitou conhecer a compreensão da equipe de enfermagem e médica sobre as metas de segurança dopaciente e destacou o papel do enfermeiro como apoiador e fomentador na promoção da segurança do paciente.

DESCRITORES: Segurança do Paciente; Equipe de Assistência ao Paciente; Serviços Médicos de Emergência.
\end{abstract}

\section{ABSTRACT}

Objective: To understand the perception of the nursing and medical staff working in an emergency unit, regarding patient safety goals. Method: This is a descriptive study with a qualitative approach, carried out in an emergency sector of a public hospital in Paraná. Data collection was carried out through interviews guided by a script, containing open and closed questions. Results: The study participants were 15 professionals, including nurses, physicians and nursing technicians, who highlighted their vision and understanding of the patient safety goals proposed by the Ministry of Health, as well as the daily application within their care practice. Conclusion: The study made it possible to know the understanding of the nursing and medical staff about patient safety goals and highlighted the role of nurses as supporters and promoters in the promotion of patient safety.

DESCRIPTORS: Patient Safety; Patient Care Team; Emergency Medical Services.

\section{RESUMEN}

Objetivo: Conocer la percepción del personal médico y de enfermería que trabaja en una unidad de urgencias, sobre las metas de seguridad del paciente. Método: Se trata de un estudio descriptivo con abordaje cualitativo, realizado en un sector de emergencia de un hospital público de Paraná. La recolección de datos se llevó a cabo a través de entrevistas guiadas por guiones, que contenían preguntas abiertas y cerradas. Resultados: Los participantes del estudio fueron 15 profesionales, entre enfermeros, médicos y técnicos de enfermería, quienes destacaron su visión y comprensión de las metas de seguridad del paciente propuestas por el Ministerio de Salud, así como la aplicación diaria dentro de su práctica asistencial. Conclusión: El estudio permitió conocer la comprensión del personal médico y de enfermería sobre las metas de seguridad del paciente y destacó el papel de las enfermeras como apoyo y promotor en la promoción de la seguridad del paciente.

DESCRIPTORES: Seguridad del Paciente, Grupo de Atención al Paciente, Servicios Médicos de Urgencia.

RECEBIDO EM: 10/06/2021 APROVADO EM: 28/06/2021

\section{Kalliny Nathiara de Oliveira Stralhoti}

Enfermeira especialista em Urgência e Emergência (FEAS). Curitiba/Paraná.

ORCID: 0000-0002-9457-3082

\section{Thais Lazaroto Roberto Cordeiro}

Enfermeira. Mestre em Ensino das Ciências da Saúde (FPP). Curitiba/Paraná.

ORCID: 0000-0002-5336-1104 


\section{Sulamita de Paula Santos}

Enfermeira. Mestre em Enfermagem (UFPR). Curitiba/Paraná.

ORCID: 0000-0003-1168-6987

\section{Luciana Aparecida Soares de Andrade}

Enfermeira. Mestre em Enfermagem (UFPR). Curitiba/Paraná.

ORCID: 0000-0002-8712-510X

\section{Juliana Euzyce Caxambu}

Enfermeira especialista em Urgência e Emergência (FEAS). Curitiba/Paraná. ORCID: 0000-0002-6726-5033

\section{INTRODUÇÃO}

$P^{S}$ egundo a Organização Mundial de Saúde (OMS) o termo segurança do paciente pode ser definido como "reduzir a um mínimo aceitável, o risco de dano desnecessário associado ao cuidado de saúde"1,2 No ano de 2004 a World Allience for Patient Safety (Aliança Mundial para a Segurança do Paciente) foi criada pela OMS, em vista dos altos números nos índices de morbi mortalidade e eventos relacionados ao atendimento ao paciente, lançando o primeiro Desafio Global para Segurança do Paciente, que foi voltado às infecções referentes a assistência à saúde. ${ }^{3,4}$

Nesse mesmo ano, no Brasil, a Agência Nacional de Vigilância Sanitária (ANVISA) inseriu em seu plano as intervenções preditas pela Aliança Mundial para a SegurançadoPaciente. ${ }^{5}$ Nesse sentido, em 2013 o Programa Nacional de Segurança do Paciente (PNSP) foi estabelecido pela Portaria GM/MSn ${ }^{\circ} 529 / 2013$, com o intuito de contribuir para a qualificação do cuidado em saúde no país. ${ }^{4,6}$

O PNSP engloba várias condutas focadas no aperfeiçoamento da segurança ambiental e gerenciamento de riscos, e tem como um dos propósitos a redução dos Eventos Adversos (EA). Um EA refere-se a uma situação que pode ser impedida e que seja dispensável, mas causou ou causaria uma injúria na estrutura ou função do corpo "incluindo lesões permanentes ou temporárias, sofrimento, morte, incapacidade ou disfunção, seja qual for a esfera do bem-estar do cliente"?

Em consonância com PNSP e visando a redução do EA, o Ministério da Saúde instituiu que os serviços de atenção à saúde implantassem as 6 Metas Internacionais de Segurança do Paciente, e publicou os Protocolos Básico de Segurança do Paciente que referem-se a elas: 1)Iden- tificação do paciente; 2) Comunicação efetiva; 3) Uso de medicamentos; 4) Cirurgia Segura; 5) Higiene das mãos para evitar infecções hospitalares; 6) Reduzir quedas e lesão por pressão ${ }^{8}$.

Os protocolos de segurança do paciente estabelecem padrões e critérios de práticas em saúde, sua importância é legitimada pela evidência de que os profissionais de saúde podem cometer erros, e esses erros podem resultar em dano grave a saúde do paciente ou até mesmo ocasionar sua morte. No intuito de impedir esse desfecho, os protocolos auxiliam na identificação precoce de situações de risco, permitindo pronta intervenção evitando o erro. ${ }^{2}$

A ocorrência de erros é uma realidade da assistência à saúde que traz consequências tanto para os pacientes, quanto para os profissionais e para organização hospitalar. ${ }^{9}$ Por reconhecer que o "errar" também está presente nos serviços de saúde cabe aos profissionais que atuam nesses serviços construir uma cultura de segurança, no sentindo de reduzir o erro por meio da identificação de situações que contribuem para sua ocorrência implementar medidas de prevenção, tornando desta forma a assistência ao paciente mais segura. ${ }^{10}$

Tendo em vista que o ambiente hospitalar é considerado como fonte de estresse devido às circunstâncias insalubres e riscos constantes nas atividades realizadas pelos profissionais, na unidade de emergência essa condição é agravada, visto que o profissional é submetido em sua rotina a uma série de situações que desencadeiam o esgotamento físico e mental, mesmo diante dessa realidade deve garantir um atendimento seguro e de qualidade. ${ }^{11}$

O setor de emergência representa um ambiente de alto risco, pois pode potencialmente ocorrer falhas, por ser considerado como uma das áreas do hospital com maior complexidade, devido a admissão de pacientes com diversos tipos de patologias e com variado grau comprometimento do seu estado de saúde, no geral trata-se de pacientes graves o que exige dos profissionais a tomada de decisão e realização de intervenções rápidas, realização de procedimentos invasivos, administração de medicações em vários níveis de complexidade e também constantemente interação entre a equipe multiprofissional, num ritmo de trabalho frenético. ${ }^{11}$

Diante disso, alguns estudos trazem a relevância da inclusão da equipe em discussões, planejamento e elaboração de novas estratégias que contribuam para o aperfeiçoamento da segurança do paciente considerando que essa participação tende a estimular os profissionais apensar e rever suas práticas e consequentemente capacitá-los por meio da disseminação de conhecimento. ${ }^{11,12}$

No atual escopo da busca pela assistência segura ao paciente é fundamental a participação a equipe no que se refere a esse tema, expressando opiniões sobre o sistema de trabalho, tornando viável tanto o reconhecimento de possíveis dificuldades, como de potenciais qualidades. ${ }^{12}$

Nesse contexto torna-se fundamental destacar a percepção da equipe multiprofissional sobre as metas de segurança do paciente, uma vez que são esses profissionais responsáveis por prestar uma assistência segura e com qualidade. Tendo o estudo como objetivo: Compreender a percepção da equipe de enfermagem e médica que atua em uma unidade de emergência, quanto às metas de segurança do paciente.

\section{MÉTODO}

Trata-se um estudo descritivo com abordagem qualitativa. A pesquisa foi realizada em um setor de emergência de um hospital público, referência em atendimento de idosos localizado em Curitiba no Paraná. 
A seleção dos participantes ocorreu por meio do convite individual, realizado pelos pesquisadores. Neste momento foram explicados o objetivo do estudo e enfatizado a importância dos resultados da pesquisa para auxiliar na melhoria do serviço. A população foi constituída por 27 profissionais, que atuam no setor de emergência do hospital estudado, entre eles médicos, enfermeiros e técnicos de enfermagem, sendo selecionados para amostra aqueles que atenderam aos seguintes critérios de inclusão da pesquisa: Trabalhar no setor de emergência do referido hospital em um dos três turnos de trabalho há pelo menos três meses; Estar em pleno vigor de suas atribuições; Concordar em participar do estudo, por meio da assinatura do Termo de Consentimento Livre e Esclarecido (TCLE).

A coleta de dados foi realizada por meio de entrevistas guiadas por um roteiro elaborado pelos pesquisadores, no período de março 2017 à junho de 2018, contendo questôes abertas e fechadas, divididas em duas partes, caracterização dos participantes e questões norteadoras que buscaram explorar o assunto pesquisado. Inicialmente foi realizado um teste piloto e não houve necessidade de alterações no instrumento de coleta.

As entrevistas foram gravadas utilizando-se gravador de voz, armazenadas em mídia digital e transcritas na íntegra em documento de texto (Microsoft Word 2016), o tempo de duração das gravações variou entre quatro e 17 minutos. Para manter o sigilo dos participantes os mesmos foram classificados com a vogal "E" (de Entrevistado) e acrescido de número conforme ordem de entrevistas, aleatórias quanto à profissão.

Para a análise dos dados foi utilizado o método de análise de conteúdo, segundo referencial teórico de Bardin. ${ }^{13} \mathrm{~A}$ análise de conteúdo trata-se de um aglomerado de recursos metodológicos aguçados que permanecem em constante desenvolvimentos, que por sua vez empregam a reflexões e raciocínios variados. ${ }^{13}$ A utilização dessa técnica permite a separação dos elementos que constituem o discurso e classificação dos fenômenos, possibilitando um entendimento mais profundo da perspectiva real da população a ser estudada. ${ }^{13}$ Os resultados obtidos foram discutidos de acordo com os achados descritos na literatura.
Todos os participantes assinaram o TCLE em cumprimento da resolução 466/12, do Ministério da Saúde, sendo a pesquisa aprovada pelo Comitê de Ética em Pesquisa da Secretaria Municipal de Saúde de Curitiba no Paraná, CAAE no 84037618.3.0000.0101, $\mathrm{n}^{\circ}$ do Parecer: 2.557.692.

\section{RESULTADOS}

Participaram do estudo 15 profissionais sendo cinco enfermeiros, cinco médicos e cinco técnicos de enfermagem. O intervalo de idades dos profissionais variou entre 25 e 52 anos, com a média de idade de 37 anos. Com relação ao sexo houve predominância do sexo feminino, com um total de 12 participantes. Quanto à formação, a maioria dos entrevistados ( $\mathrm{n}=12$ ) tinham pelo menos uma especialização e apenas três técnicos de enfermagem não possuíam formação adicional.

\section{Compreensão da equipe quanto à segurança do paciente no setor de emergência}

Identificou-se que os participantes compreendem o termo "segurança do paciente" como a redução de danos durante $o$ atendimento ou internamento, visando o bem- estar e melhora do estado de saúde do doente. Também atribuem segurança a qualidade da assistência prestada.

E1: A se eu tentar conceituar, acho
que são procedimentos e condutas que
visam reduzir os eventuais danos que
podem acontecer dentro do atendi-
mento em saúde, algo do tipo.
E5 - São metas e formas de tentativa
da equipe em vetar o máximo de da-
nos possiveis durante o internamento
desse paciente.

Alguns entrevistados atribuem a segurança do paciente a medidas de prevenção e citaram exemplos de açóes que podem ser realizadas no setor de emergência visando à segurança do paciente, como prevenção de quedas e erros de medicação:

$$
\begin{aligned}
& \text { E3 - Segurança do paciente para } \\
& \text { mim é prevenir quedas, erros de }
\end{aligned}
$$

medicação, qualquer dano para o paciente que seja irreversivel ou reversivel, mas com custo elevado para mim à segurança do paciente é a prevenção, açôes para prevenir.

E6 - Segurança do paciente seria na hora de fazer a medicação, do risco de quedas do paciente, da comunicação entre profissionais do setor da equipe de enfermagem e dos médicos também, dasprescrições, tudo identificado, então tudo que venha a promover a saúde do paciente, tudo que não cause riscos.

Por meio das falas dos participantes foi possível perceber que a equipe está ciente da importância do seu papel no processo de segurança do paciente e de que essa atenção deve permanecer durante todo o período de internação do cliente contribuindo para a qualidade da assistência. Porém nos chama a atenção o fato de que alguns profissionais da área médica atribuem a responsabilidade pela segurança do paciente à equipe de enfermagem, assim como o cumprimento das metas de segurança como identificação do paciente e prevenção de quedas:

E8 - [...] é que a gente da parte médica não é tão cobrado assim, acho que mais a enfermagem mesmo, mas eu sei que elas são cobradas de checagem de medicação, eu acho que é uma das metas de segurança, higiene das mãos que nos somos muito cobrados também quanto a isso e identificação do paciente também ébem cobrado e bem seguido assim, [...] isso tem tudo em um documentação que a enfermeiras geralmente lêem junto com os familiares e os familiares assinam pra ajudarem a cuidar dessa parte também pra evitar acidentes com os pacientes.

E13 - Eu vejo, o que mais vejo aqui na emergência do risco de quedas, eu vejo sistematicamente as enfermeiras implementando mesmo, bom a identificação com certeza porque todas ganham a pulseirinha logo que chegam [...]

\section{DISCUSSÃO}

Por meio da análise das falas dos parti- 
cipantes do estudo, foi possível conhecer a compreensão da equipe médica e de enfermagem que atua no setor de emergência sobre o tema segurança do paciente em um escopo geral incluindo além do conhecimento dos conceitos básicos sobre o assunto, as suas sugestões e contribuições para melhoria da segurança do paciente em seu ambiente de trabalho e as dificuldades que eles enfrentam para cumprimento dos protocolos institucionais, que foram organizados nas quatro categorias identificadas neste estudo.

A compreensão da equipe quanto à segurança do paciente estão em conformidade aos encontrados na literatura, pois os participantes compreendem o conceito de segurança do paciente como sendo a assistência segura e a minimização de danos causados ao paciente. Segundo a OMS a segurança do paciente pode ser conceituada como a redução do risco de da- nos desnecessários associados às intervenções em saúde até um mínimo aceitável. ${ }^{14}$

Foi perceptível ao longo das entrevistas que parte significativa dos profissionais não sabia dizer quais são as seis metas de segurança do paciente estabelecidas, sendo que apenas dois souberam citá-las. Porém, durante as suas falas sobre o conceito de segurança do paciente, identificou-se alguns exemplos de ações que são realizadas em sua rotina que são condizentes e estão associadas às metas internacionais de segurança do paciente. ${ }^{15}$

Com relação ao papel do enfermeiro várias vezes citado, fica evidente sua importância no que se refere à organização do processo de trabalho da equipe, pois cabe a esse profissional a viabilização de condições seguras e de qualidade, envolvendo o espaço físico do setor, os materiais disponibilizados e a qualificação da equipe. Além disso, o enfermeiro tem papel ativo no acompanhamento das atividades dos técnicos de enfermagem que permite a detecção e correção prévia de situaçôes que poderiam desencadear um dano desnecessário ao paciente. Todos esses fatores refletem na assistência segura. ${ }^{16}$

\section{CONCLUSÃO}

O estudo possibilitou conhecer a compreensão da equipe de enfermagem e médica sobre as metas de segurança do paciente e evidenciou que estes profissionais estão atentos ao assunto e reconhecem sua importância para qualidade da assistência ao paciente. Apesar da maioria dos participantes não conhecerem os protocolos de segurança do paciente da instituição eles sabiam do que se tratava e demonstram interesse em aprender mais sobre o tema. Destaca-se o papel do enfermeiro no contexto da promoção da segurança do paciente.

\section{REFERÊNCIAS}

1. Kohn LT.; Corrigan JM.; Donaldson MS. To err is human: building a safer health system. Committe on Quality of Health Care in America, Institute of Medicine. Whashington, DC: National Academy Press; 2000.

2. Ministério da Saúde (BR), Agência Nacional de Vigilância Sanitária, Fundação Oswaldo Cruz. Documento de referência para o Programa Nacional de Segurança do Paciente. Brasília: Ministério da Saúde, 2014. p. 7.

3. Fonseca AS; Peterline FL; Costa DA. Segurança do Paciente. São Paulo: Martinari; 2014.

4. Ministério da Saúde (BR), Portal da Saúde. Programa Nacional de Segurança do Paciente. Brasília: Ministério da Saúde, 2017.

5. Ministério da Saúde (BR), Agência Nacional de Vigilância Sanitária. Implantação do Núcleo de Segurança do Paciente em Serviços de Saúde - Série Segurança do Paciente e Qualidade em Serviços de Saúde. Brasília: Ministério da Saúde, 2016.

6. Oliveira PP. Desafios da Segurança do Paciente e a Qualidade em Serviços de Oncologia. Rev. enferm. Cent.-Oeste Min. 2017 [citado em 2018 fev. 25];7. Disponível em: http://dx.doi. org/10.19175/recom.v7i0.2692

7. Souza RF, Alves AS, Alencar IGM. Eventos adversos na unidade de terapia intensiva. Rev. enferm. UFPE on line. 2018 [citado em 2018 jan. 29];12(1). p. 20. Disponivel em: https://doi. org/10.5205/1981-8963-v12i1a25205p19-27-2018

8. Ministério da Saúde (BR), Agência Nacional de Vigilância Sanitária. Como posso contribuir para aumentar a segurança do paciente?. Brasília: Ministério da Saúde, 2017.

9. Siman AG, Brito MJM. Mudanças na prática de enfermagem para melhorar a segurança do paciente. Rev. Gaúcha Enferm.
2017 [citado em 2018 dez. 22];37(spe). Disponível em: http:// dx.doi.org/10.1590/1983-1447.2016.esp.68271

10. Duarte SCM, Stipp MAC, Silva MM, Oliveira FT. Eventos adversos e segurança na assistência de enfermagem. Rev Bras Enferm. 2015 [citado em 2018 jan. 29];68(1). Disponivel em: http:// dx.doi.org/10.1590/0034-7167.2015680120p

11. Källberg AS, Ehrenberg A, Florin J, Östergren J, Göransson KE. Physicians' and nurses' perceptions of patient safety risks in the emergency department. Int Emerg Nurs. 2017 [citado em 2018 jan. 04];33. Disponível em: http://dx.doi.org/10.1016/j. ienj.2017.01.002

12. Borges F, Bohrer CD, Kawamoto AM, Oliveira JLC, Nicola AL. Grau da cultura de segurança do paciente na percepção da equipe multiprofissional hospitalar. Revista Varia Scientia - Ciências da Saúde. [citado em 2018 jan. 26];2(1). Disponível em: http://saber. unioeste.br/index.php/variasaude/article/view/14293/10053

13. Bardin L. Análise de Conteúdo. Lisboa: Edições 70; 2009.

14. Organização Mundial Da Saúde. The Conceptual Framework for the International Classification for Patient Safety. Geneva: WHO; 2009.

15. Joint Comission International. Padrões de Acreditação da Joint Comission International para Hospitais. Tradução Oficial para o Português, da quarta edição do original "Joint Comission International Accreditation Standards for Hospitals". Illinois: Department of Publications; 2011.

16. Dias JD, Mekaro KS, Tibes CMS, Zem-Mascarenhas SH. Compreensão de enfermeiros sobre segurança do paciente e erros de medicação. REME rev. min. enferm. [citado em 2018 jan. 14];18(4). Disponivel em: http://www.dx.doi.org/10.5935/14152762.20140064 